\title{
Mass splittings of SU(3) baryons within a chiral soliton model
}

\author{
Ghil-Seok YANG ${ }^{1 *)}$ and Hyun-Chul $\mathrm{KIM}^{2,3,4 * *)}$ \\ ${ }^{1}$ Center for High Energy Physics $\&$ Department of Physics, Kyungpook National \\ University, Daegu 702-701, Republic of Korea \\ ${ }^{2}$ Department of Physics, Inha University, Incheon 402-751, Republic of Korea \\ ${ }^{3}$ Department of Physics, University of Connecticut, Storrs, CT 06269, U.S.A. \\ ${ }^{4}$ School of Physics, Korea Institute for Advanced Study, Seoul 130-722, Republic of \\ Korea
}

\begin{abstract}
Considering simultaneously isospin and SU(3) flavor symmetry breakings, we investigate the complete mass splittings of SU(3) baryons within a chiral soliton model, a "modelindependent approach" being employed. In linear order several new mass relations are derived, which are mostly generalizations of existing mass formulae. The dynamical quantities appearing in the expressions for the masses are fixed by fitting them to the masses of the baryon octet and those of $\Omega^{-}$and $\Theta^{+}$as input rather than by extracting them from a calculated self-consistent soliton profile. In particular, the consideration of isospin symmetry breaking allows us to use the experimental data of the whole octet baryon masses as input. We predict the masses of the baryon decuplet and antidecuplet without any further adjustable free parameter. In addition, we also obtain the pion-nucleon sigma term which turns out to be $\Sigma_{\pi N}=36.4 \pm 3.9 \mathrm{MeV}$. We get the ratio of the current light quark masses $R=58.1 \pm 1.3$. The present results indicate that the recent experimental data for the $\Theta^{+}(1524)$ are compatible with the experimental data of the octet and decuplet masses.
\end{abstract}

\section{$\S 1$. Introduction}

The mass splittings of $\mathrm{SU}(3)$ baryons are the first observables that any lowenergy effective model for quantum chromodynamics (QCD) should explain. Since the Skyrme model was suggested as a topological effective model of QCD in the large $N_{c}$ limit, the picture of the topological and particularly non-topological chiral soliton has been known to be very successful in describing the splitting between the baryon octet and decuplet. One of the most interesting features in this picture is that the low-lying baryons can be regarded as rotational excitations of the chiral soliton: The baryon octet (8) appear as the lowest representation with spin $1 / 2$ and positive parity. The baryon decuplet (10) with spin $3 / 2$ arises from the next rotational excitation of the chiral soliton. When we proceed further with these excitations, we find that there are baryon antidecuplet $(\overline{\mathbf{1 0}})$, eikosiheptaplet $(\mathbf{2 7})$, and so on. In particular, the baryon antidecuplet is the first excitation consisting of exotic pentaquark baryons (1), (2), (3) which have attracted much attention, since the LEPS collaboration announced the first measurement of the pentaquark baryon $\Theta^{+}(\underline{4})$ However, a series of the CLAS experiments has reported null results of the $\Theta^{+(5),(6), 7),(8)}$ and has casted doubt on its existence. On the other hand, the DIANA collaboration has continued to search for the $\Theta^{+(9),(10)}$ and announced very recently

\footnotetext{
*) E-mail: ghsyang@knu.ac.kr

**) E-mail: hchkim@inha.ac.kr
} 
the formation of a narrow $p K^{0}$ peak with mass of $1538 \pm 2 \mathrm{MeV} / c^{2}$ and width of $\Gamma=0.39 \pm 0.10 \mathrm{MeV}$ in the $K^{+} n \rightarrow K^{0} p$ reaction with higher statistical significance $(6 \sigma-8 \sigma) \stackrel{10}{10}$ Moreover, other new positive experiments for the $\Theta^{+}$have been reported (11), 12), 13), (14) The LEPS collaboration also reported the evidence of the $\Theta^{+}$ existence. $\left.{ }^{15}\right) \quad M_{\Theta}=1.524 \pm 0.002 \pm 0.003 \mathrm{GeV} / c^{2}$ with the statistical significance $5.1 \sigma$. The peak position is shifted by $+3 \mathrm{MeV}$ systematically due to the minimum momentum spectator approximation.

In addition to the $\Theta^{+}$baryon, the GRAAL experiment $\left(\frac{16), 17), 18)}{16}\right.$ has found a new nucleon-like resonance around $1.67 \mathrm{GeV}$ from $\eta$ photoproduction off the deutron in the neutron channel. The decay width was measured to be around $40 \mathrm{MeV}$. However, Fermi-motion being effects excluded, this decay width may decrease ${ }^{\text {(19) }}$ References ${ }^{16)}$, (17), 18) have shown that the resonant structure was not seen in the quasi-free proton channel. Note that this newly found $N^{*}$ resonance is consistent with the theoretical predictions 20 , 210 of non-strange exotic baryons. Moreover, the narrow width and its dependence on the initial isospin state are the typical characteristics for the photo-excitation of the non-strange antidecuplet pentaquark. 22),23) Very recently, a new analysis of the free proton GRAAL data 24 , (25), (26), 27), 28) has revealed a resonance structure with a mass around $1685 \mathrm{MeV}$ and width $\Gamma \leq 15$ $\mathrm{MeV}$. However, we have to mention that the results of Ref.24) do not agree with those of Ref. $\left.{ }^{[29}\right)$ For a detailed discussion of this discrepancy, we refer to Ref.(25) The CB-ELSA collaboration ${ }^{30}$ ) has also announced an evidence for this newly found $N^{*}$ resonance in line with that of GRAAL. All these experimental facts are consistent with the results for the transition magnetic moments in the $\chi \mathrm{QSM}^{222}$, 23) and phenomenological analysis for the non-strange pentaquark baryons. ${ }^{\text {[31) }}$ Based on these results, theoretical calculations of the $\gamma N \rightarrow \eta N$ reaction ${ }^{32}$, 33) describe qualitatively well the GRAAL data. In Refs. 21), 34) the non-strange partners of the $\Theta^{+}$were also studied, results of which are comparable with those in this work.

In the original predictions of the $\Theta^{+}$mass from a chiral soliton model $\left.(\chi \mathrm{SM})^{2}\right)$ the theoretical analyses are partially based on specific model calculations, ${ }^{35)}, 36$ while some dynamical parameters are fixed by the experimental masses of the baryon octet and decuplet and the empirical value of the $\pi N$ sigma term $\Sigma_{\pi N}$. In particular, the second moment of inertia ${ }^{37}$, ,38) of the chiral soliton known as $I_{2}$, which is an essential quantity to determine the shift of the antidecuplet center from the octet center in the chiral limit, is given only in a wide range: $0.43 \mathrm{fm}<I_{2}<0.55 \mathrm{fm}$, depending on specific models such as either the Skyrme mode ${ }^{39}$, 40 ) or the chiral quark-soliton model $(\chi \mathrm{QSM}) \stackrel{[37), 38)}{\text {,36 }}$ Thus, some of model-dependent uncertainties are inherent in previous analyses of the $\mathrm{SU}(3)$ baryon masses.

In the present work, we aim at determining the masses of the baryon decuplet, and a part of those of the baryon antidecuplet with all dynamical parameters fixed to existing data for the masses of the baryon octet. 41) In order to incorporate these data, however, it is essential to consider the breakdown of isospin symmetry, since the experimental data involve already the effects of isospin symmetry breaking that consist of two different contributions: The electromagnetic (EM) and hadronic ones. The isospin breaking effects due to the EM corrections were already investigated in Ref.42) within the framework of the $\chi \mathrm{SM}$. In the present work, we will intro- 
duce additionally the hadronic contributions of isospin symmetry breaking to the mass splittings, so that we can analyze the mass splittings of the $\mathrm{SU}(3)$ baryons consistently.

Having taken into account these effects of isospin symmetry breaking, we are able to fix unequivocally the relevant model parameters by employing the experimental data of the baryon octet masses. In addition, we will use the experimental values of the $\Theta^{+}(1524)$, though its existence is disputable, as well as of the $N^{*}(1685)$ such that we can determine $I_{2}$ unambiguously. We will show that the masses of the baryon decuplet and parts of the antidecuplet are determined uniquely without any adjustable parameters.

The present work is sketched as follows: In Section II, we describe the general formalism of the present approach for the mass splittings of the $\mathrm{SU}(3)$ baryons. We also discuss various relations between $\mathrm{SU}(3)$ baryon masses such as the generalized relations of Gell-Mann-Okubo, of Coleman-Glashow relation, and of Guadagnini. In Section III, we show how to determine the dynamical parameters of the $\chi \mathrm{SM}$, using the existing experimental data for the octet with $\Omega^{-}(1672)$ and $\Theta^{+}(1524)$ masses. We present the final results of the decuplet and antidecuplet masses. In the last Section, we summarize the present work and draw conclusions.

\section{$\S 2 . \quad$ General Formalism}

The mass splittings of SU(3) baryons within a chiral soliton have been extensively studied in Refs. (2), 43) As mentioned in the previous Section, the effects of isospin symmetry breaking arise from two different sources, i.e. the mass differences of the up and down quarks and the EM interactions. The effects of isospin symmetry breaking on the baryon mass splittings have been studied in Refs. ${ }^{44}$, (45) within the $\chi$ QSM. The EM mass splittings of the SU(3) baryons have been already investigated in Ref. ${ }^{42}$ Thus, we briefly review how to construct the collective Hamiltonians for the masses of the $\mathrm{SU}(3)$ baryons with isospin symmetry breaking taken into account in the present Section.

\subsection{Collective Hamiltonian and $S U(3)$ baryon states}

We start from the collective Hamiltonian in the $\mathrm{SU}(3) \chi \mathrm{SM}:[38), 37)$

$$
H=M_{\mathrm{cl}}+H_{\mathrm{rot}}+H_{\mathrm{sb}},
$$

where $M_{\mathrm{cl}}$ denotes the classical soliton mass. The $H_{\text {rot }}$ and $H_{\mathrm{sb}}$ respectively stand for the $1 / N_{c}$ rotational and symmetry-breaking corrections including the effects of isospin and $\mathrm{SU}(3)_{\mathrm{f}}$ symmetry breakings $\cdot \frac{45)}{}$

$$
\begin{gathered}
H_{\text {rot }}=\frac{1}{2 I_{1}} \sum_{i=1}^{3} \hat{J}_{i}^{2}+\frac{1}{2 I_{2}} \sum_{p=4}^{7} \hat{J}_{p}^{2} \\
H_{\mathrm{sb}}=\left(m_{\mathrm{d}}-m_{\mathrm{u}}\right)\left(\frac{\sqrt{3}}{2} \alpha D_{38}^{(8)}(\mathcal{R})+\beta \hat{T}_{3}+\frac{1}{2} \gamma \sum_{i=1}^{3} D_{3 i}^{(8)}(\mathcal{R}) \hat{J}_{i}\right)
\end{gathered}
$$




$$
\begin{aligned}
& +\left(m_{\mathrm{s}}-\hat{m}\right)\left(\alpha D_{88}^{(8)}(\mathcal{R})+\beta \hat{Y}+\frac{1}{\sqrt{3}} \gamma \sum_{i=1}^{3} D_{8 i}^{(8)}(\mathcal{R}) \hat{J}_{i}\right) \\
& +\left(m_{u}+m_{d}+m_{s}\right) \sigma,
\end{aligned}
$$

where $I_{1,2}$ represent the soliton moments of inertia that depend on dynamics of specific formulations of the $\chi \mathrm{SM}$. The $J_{i}$ denote the generators of the $\mathrm{SU}(3)$ group. The $m_{\mathrm{u}}, m_{\mathrm{d}}$, and $m_{\mathrm{s}}$ designate the up, down, and strange current quark masses, respectively. The $\hat{m}$ is the average of the up and down quark masses. The $D_{a b}^{(\mathcal{R})}(\mathcal{R})$ indicate the SU(3) Wigner $D$ functions. The $\hat{Y}$ and $\hat{T}_{3}$ are the operators of the hypercharge and isospin third component, respectively. The $\alpha, \beta$, and $\gamma$ are given in terms of the $\pi N$ sigma term $\Sigma_{\pi N}$ and soliton moments of inertia $I_{1,2}$ and $K_{1,2}$ as follows:

$$
\alpha=-\left(\frac{2}{3} \frac{\Sigma_{\pi N}}{m_{\mathrm{u}}+m_{\mathrm{d}}}-\frac{K_{2}}{I_{2}}\right), \quad \beta=-\frac{K_{2}}{I_{2}}, \quad \gamma=2\left(\frac{K_{1}}{I_{1}}-\frac{K_{2}}{I_{2}}\right) .
$$

Since $\alpha, \beta$, and $\gamma$ depend on the moments of inertia, they are also related to details of specific dynamics of the $\chi \mathrm{SM}$. Note that $\alpha, \beta$, and $\gamma$ defined in the present work do not contain the strange quark mass, while those in Refs. $\left.{ }^{(2)}, 43\right)$ include it. The $\sigma$ is proportional to the $\Sigma_{\pi N}$ as follows:

$$
\sigma=-(\alpha+\beta)=\frac{2}{3} \frac{\Sigma_{\pi N}}{m_{\mathrm{u}}+m_{\mathrm{d}}}
$$

which can be absorbed by the center of the mass splittings from the rotational Hamiltonian $H_{\text {rot }}$.

In the $\chi \mathrm{SM}$, there is a very important constraint for the collective quantization:

$$
J_{8}=-\frac{N_{c}}{2 \sqrt{3}} B=-\frac{\sqrt{3}}{2}, \quad Y^{\prime}=\frac{2}{\sqrt{3}} J_{8}=-\frac{N_{c}}{3}=-1,
$$

where $B$ is the baryon number. It is related to the eighth component of the soliton angular velocity that is due to the presence of the discrete valence quark level in the Dirac-sea spectrum in the $\mathrm{SU}(3) \chi \mathrm{SM}, \frac{38}{(36), 46}$ while it arises from the Wess-Zumino term in the SU(3) Skyrme model (47), (48), (49) Its presence has no effects on the chiral soliton but allows us to take only the $\mathrm{SU}(3)_{\mathrm{f}}$ irreducible representations with zero triality. Thus, the allowed $\mathrm{SU}(3)_{\mathrm{f}}$ multiplets are the baryon octet $(J=1 / 2)$, decuplet $(J=3 / 2)$, and antidecuplet $(J=1 / 2)$, etc. In the representation $(p, q)$ of the $\mathrm{SU}(3)$ group, we can have the following relation:

$$
\sum_{i=1}^{8} J_{i}^{2}=\frac{1}{3}\left[p^{2}+q^{2}+p q+3(p+q)\right],
$$

which yields the eigenvalues of the rotational collective Hamiltonian $H_{\text {rot }}$ in Eq. (2.2) as follows:

$$
E_{(p, q), J}=\mathcal{M}_{\mathrm{cl}}+\frac{1}{2}\left(\frac{1}{I_{1}}-\frac{1}{I_{2}}\right) J(J+1)
$$




$$
+\frac{1}{6 I_{2}}\left(p^{2}+q^{2}+3(p+q)+p q\right)-\frac{3}{8 I_{2}} .
$$

The allowed SU(3) baryon multiplets with zero triality are given as

$$
\begin{aligned}
& (p, q)=(1,1) \rightarrow J=1 / 2 \text { (octet) }, \\
& (p, q)=(3,0) \rightarrow J=3 / 2 \text { (decuplet) } \\
& (p, q)=(0,3) \rightarrow J=1 / 2 \text { (antidecuplet). }
\end{aligned}
$$

Thus, the mass splittings between the centers of the multiplets are obtained as follows:

$$
\begin{aligned}
\Delta \bar{M}_{\mathbf{1 0}-\mathbf{8}} & =E_{(3,0), J=3 / 2}-E_{(1,1), J=1 / 2}=\bar{M}_{\mathbf{1 0}}-\bar{M}_{\mathbf{8}}=\frac{3}{2 I_{1}} \\
\Delta \bar{M}_{\overline{\mathbf{1 0}}-\mathbf{8}} & =E_{(0,3), J=1 / 2}-E_{(1,1), J=1 / 2}=\bar{M}_{\overline{\mathbf{1 0}}}-\bar{M}_{\mathbf{8}}=\frac{3}{2 I_{2}} \\
\Delta \bar{M}_{\overline{\mathbf{1 0}}_{-\mathbf{1 0}}}=E_{(0,3), J=1 / 2}-E_{(3,0), J=3 / 2} & =\bar{M}_{\overline{\mathbf{1 0}}}-\bar{M}_{\mathbf{1 0}}=-\frac{3}{2 I_{1}}+\frac{3}{2 I_{2}}
\end{aligned}
$$

which shows that they arise from the rotational excitations. It is well known and understood that in the $\chi \mathrm{SM}$ one cannot calculate the absolute values of baryonic masses unless one incorporates some non-relativistic corrections. ${ }^{50}$ ) We do not do this in the present work and concentrate rather on the mass splittings, which are all well defined. Thus, it is crucial to determine the soliton moments of inertia $I_{1,2}$ uniquely. In all $\chi \mathrm{SM}$ calculations, $I_{1}$ turns out to be larger than $I_{2}$, which leads to the consequence that the masses of the antidecuplet become larger than those of the octet.

In order to determine the $\mathrm{SU}(3)$ baryon mass splittings, we now consider the symmetry-breaking Hamiltonian $H_{\mathrm{sb}}$ in Eq. (2.3). The corrections due to the $\mathrm{SU}(3)_{\mathrm{f}}$ and isospin symmetry breaking effects are obtained perturbatively by calculating the matrix elements of the $H_{\mathrm{sb}}$ between the diagonal baryon states that are written as the $\mathrm{SU}(3)$ Wigner $D$ functions in representation $\mathcal{R}$ :

$$
\begin{aligned}
& \left\langle A \mid \mathcal{R}, B\left(Y T T_{3}, Y^{\prime} J J_{3}\right)\right\rangle \\
= & \Psi_{\left(\mathcal{R}^{*} ; Y^{\prime} J J_{3}\right)}^{\left(\mathcal{R} ; Y T J_{3}\right)}(A)=\sqrt{\operatorname{dim}(\mathcal{R})}(-)^{J_{3}+Y^{\prime} / 2} D_{\left(Y, T, T_{3}\right)\left(-Y^{\prime}, J,-J_{3}\right)}^{(\mathcal{R}) *}(A),
\end{aligned}
$$

where $\mathcal{R}$ stands for the allowed irreducible representations of the $\mathrm{SU}(3)_{\mathrm{f}}$ group, i.e. $\mathcal{R}=8,10, \overline{10}, \cdots$ and $Y, T, T_{3}$ are the corresponding hypercharge, isospin, and its third component, respectively. The constraint of the right hypercharge $Y^{\prime}=1$ selects a tower of allowed $\mathrm{SU}(3)_{\mathrm{f}}$ representations: The lowest ones, that is, the baryon octet and decuplet, coincide with those of the quark model. This has been considered as a success of the collective quantization and as a sign of certain duality between rigidly rotating heavy soliton and constituent quark model. The third lowest representation is the antidecuplet which has been considered as an artifact of the model and therefore disregarded until the work of Diakonov et al ${ }^{(2)}$

Since the symmetry-breaking term of the collective Hamiltonian in Eq. (2.3) mixes different $\mathrm{SU}(3)_{\mathrm{f}}$ representations, the collective wave functions are no more in pure states but are given as the following linear combinations:

$$
\left|B_{8}\right\rangle=\left|8_{1 / 2}, B\right\rangle+c \frac{B}{10}\left|\overline{10}_{1 / 2}, B\right\rangle+c_{27}^{B}\left|27_{1 / 2}, B\right\rangle,
$$




$$
\begin{aligned}
& \left|B_{10}\right\rangle=\left|10_{3 / 2}, B\right\rangle+a_{27}^{B}\left|27_{3 / 2}, B\right\rangle+a_{35}^{B}\left|35_{3 / 2}, B\right\rangle, \\
& \left|B_{\overline{10}}\right\rangle=\left|\overline{10}_{1 / 2}, B\right\rangle+d_{8}^{B}\left|8_{1 / 2}, B\right\rangle+d_{27}^{B}\left|27_{1 / 2}, B\right\rangle+d \frac{B}{35}\left|\overline{35}_{1 / 2}, B\right\rangle,
\end{aligned}
$$

where $\left|B_{\mathcal{R}}\right\rangle$ denotes the state which reduces to the $\mathrm{SU}(3)_{\mathrm{f}}$ representation $\mathcal{R}$ in the formal limit $m_{s} \rightarrow 0$. Here, the spin indices $J_{3}$ have been suppressed. The $m_{s^{-}}$ dependent (through the linear $m_{s}$ dependence on $\alpha, \beta$ and $\gamma$ ) coefficients in Eq.(2.12) read:

$$
\begin{aligned}
& c_{10}^{B}=c_{\overline{10}}\left[\begin{array}{c}
\sqrt{5} \\
0 \\
\sqrt{5} \\
0
\end{array}\right], c_{27}^{B}=c_{27}\left[\begin{array}{c}
\sqrt{6} \\
3 \\
2 \\
\sqrt{6}
\end{array}\right], a_{27}^{B}=a_{27}\left[\begin{array}{c}
\sqrt{15 / 2} \\
2 \\
\sqrt{3 / 2} \\
0
\end{array}\right], a_{35}^{B}=a_{35}\left[\begin{array}{c}
5 / \sqrt{14} \\
2 \sqrt{5 / 7} \\
3 \sqrt{5 / 14} \\
2 \sqrt{5 / 7}
\end{array}\right], \\
& d_{8}^{B}=d_{8}\left[\begin{array}{c}
0 \\
\sqrt{5} \\
\sqrt{5} \\
0
\end{array}\right], \quad d_{27}^{B}=d_{27}\left[\begin{array}{c}
0 \\
\sqrt{3 / 10} \\
2 / \sqrt{5} \\
\sqrt{3 / 2}
\end{array}\right], \quad d \frac{B}{35}=d_{\overline{35}}\left[\begin{array}{c}
1 / \sqrt{7} \\
3 /(2 \sqrt{14)} \\
1 / \sqrt{7} \\
\sqrt{5 / 56}
\end{array}\right],
\end{aligned}
$$

respectively in the basis $[N, \Lambda, \Sigma, \Xi],\left[\Delta, \Sigma^{*}, \Xi^{*}, \Omega\right],\left[\Theta^{+}, N_{\overline{10}}, \Sigma_{\overline{10}}, \Xi_{\overline{10}}\right]$ and analogous states in $\mathcal{R}=27,35, \overline{35}$, and

$$
\begin{array}{ll}
c_{\overline{10}}=-\frac{I_{2}}{15}\left(m_{s}-\hat{m}\right)\left(\alpha+\frac{1}{2} \gamma\right), & c_{27}=-\frac{I_{2}}{25}\left(m_{s}-\hat{m}\right)\left(\alpha-\frac{1}{6} \gamma\right), \\
a_{27}=-\frac{I_{2}}{8}\left(m_{s}-\hat{m}\right)\left(\alpha+\frac{5}{6} \gamma\right), & a_{35}=-\frac{I_{2}}{24}\left(m_{s}-\hat{m}\right)\left(\alpha-\frac{1}{2} \gamma\right), \\
d_{8}=\frac{I_{2}}{15}\left(m_{s}-\hat{m}\right)\left(\alpha+\frac{1}{2} \gamma\right), & d_{27}=-\frac{I_{2}}{8}\left(m_{s}-\hat{m}\right)\left(\alpha-\frac{7}{6} \gamma\right), \\
d_{\overline{35}}=-\frac{I_{2}}{4}\left(m_{s}-\hat{m}\right)\left(\alpha+\frac{1}{6} \gamma\right) . &
\end{array}
$$

We will show later that these mixing coefficients are determined uniquely in the present scheme.

\subsection{Electromagnetic corrections to $S U(3)$ baryon masses}

The EM mass corrections to $\mathrm{SU}(3)$ baryon masses were already discussed in Ref.42) However, since they consist of an essential part of the present analysis, we will recapitulate them in this subsection. The following baryonic two-point correlation functions of the EM current will provide the EM mass corrections:

$$
M_{B}^{\mathrm{EM}}=\frac{1}{2} \int d^{3} x d^{3} y\left\langle B\left|T\left[J_{\mu}(\boldsymbol{x}) J^{\mu}(\boldsymbol{y})\right]\right| B\right\rangle D_{\gamma}(\boldsymbol{x}, \boldsymbol{y})=\left\langle B\left|\mathcal{O}^{\mathrm{EM}}\right| B\right\rangle,
$$

where $J^{\mu}$ is defined as $J^{\mu}(x)=e \bar{\psi}(x) \gamma_{\mu} \hat{Q} \psi(x)$ with the electric charge $e$ and the quark charge operator $\hat{Q}$ defined as the Gell-Mann-Nishijima relation $\hat{Q}=$ $\left(\lambda_{3}+\lambda_{8} / \sqrt{3}\right) / 2$. The $D_{\gamma}$ denotes a static photon propagator which will be absorbed in parameters we will fit to experimental data. Using the fact that the EM current is taken as an octet operator, we write the most general form of the $\mathcal{O}_{\mathrm{EM}}$ as a collective 
operator

$$
\mathcal{O}^{\mathrm{EM}}=\alpha_{1} \sum_{i=1}^{3} D_{Q i}^{(8)} D_{Q i}^{(8)}+\alpha_{2} \sum_{p=4}^{7} D_{Q p}^{(8)} D_{Q p}^{(8)}+\alpha_{3} D_{Q 8}^{(8)} D_{Q 8}^{(8)}
$$

where $D_{Q a}^{(8)}=\left(D_{3 a}^{(8)}+D_{8 a}^{(8)} / \sqrt{3}\right) / 2$. The parameters $\alpha_{i}$ depend on specific dynamics of a $\chi \mathrm{SM}$, which will be fitted to the empirical data of the EM mass differences. The product of two octet operators can be expanded in terms of irreducible operators $\mathbf{1} \oplus \mathbf{8}_{\mathbf{s}} \oplus \mathbf{8}_{\mathbf{a}} \oplus \mathbf{1 0} \oplus \overline{\mathbf{1 0}} \oplus \mathbf{2 7}$. Note, however, that because of Bose symmetry we are left only with the singlet, the octet, and the eikosiheptaplet, which are all symmetric. We rewrite $\mathcal{O}^{\mathrm{EM}}$ in terms of a new set of parameters $c^{(n)}$ as follows:

$$
\begin{aligned}
\mathcal{O}^{\mathrm{EM}} & =c^{(27)}\left(\sqrt{5} D_{\Sigma_{2}^{0} \Lambda_{27}}^{(27)}+\sqrt{3} D_{\Sigma_{1}^{0} \Lambda_{27}}^{(27)}+D_{\Lambda_{27} \Lambda_{27}}^{(27)}\right) \\
& +c^{(8)}\left(\sqrt{3} D_{\Sigma^{0} \Lambda}^{(8)}+D_{\Lambda \Lambda}^{(8)}\right)+c^{(1)} D_{\Lambda \Lambda}^{(1)} .
\end{aligned}
$$

The last term in Eq.(2.17) does not contribute to the mass splittings. The EM mass can be obtained by sandwiching the collective operator $\mathcal{O}_{\text {EM }}$ in Eq.(2.16) between the baryon states. The corresponding results can be written for the baryon octet

$$
\begin{aligned}
& M_{N}^{\mathrm{EM}}=\frac{1}{5}\left(c^{(8)}+\frac{4}{9} c^{(27)}\right) T_{3}+\frac{3}{5}\left(c^{(8)}+\frac{2}{27} c^{(27)}\right)\left(T_{3}^{2}+\frac{1}{4}\right)+c^{(1)}, \\
& M_{\Lambda}^{\mathrm{EM}}=\frac{1}{10}\left(c^{(8)}-\frac{2}{3} c^{(27)}\right)+c^{(1)}, \\
& M_{\Sigma}^{\mathrm{EM}}=\frac{1}{2} c^{(8)} T_{3}+\frac{2}{9} c^{(27)} T_{3}^{2}-\frac{1}{10}\left(c^{(8)}+\frac{14}{9} c^{(27)}\right)+c^{(1)}, \\
& M_{\Xi}^{\mathrm{EM}}=\frac{4}{5}\left(c^{(8)}-\frac{1}{9} c^{(27)}\right) T_{3}-\frac{2}{5}\left(c^{(8)}-\frac{1}{9} c^{(27)}\right)\left(T_{3}^{2}+\frac{1}{4}\right)+c^{(1)},
\end{aligned}
$$

and for the baryon decuplet

$$
\begin{aligned}
& M_{\Delta}^{\mathrm{EM}}=\frac{1}{4}\left(c^{(8)}+\frac{8}{63} c^{(27)}\right) T_{3}+\frac{5}{63} c^{(27)} T_{3}^{2}+\frac{1}{8}\left(c^{(8)}-\frac{2}{3} c^{(27)}\right)+c^{(1)}, \\
& M_{\Sigma^{*}}^{\mathrm{EM}}=\frac{1}{4}\left(c^{(8)}-\frac{4}{21} c^{(27)}\right) T_{3}+\frac{5}{63} c^{(27)}\left(T_{3}^{2}-1\right)+c^{(1)}, \\
& M_{\Xi^{*}}^{\mathrm{EM}}=\frac{1}{4}\left(c^{(8)}-\frac{32}{63} c^{(27)}\right) T_{3}-\frac{1}{4}\left(c^{(8)}+\frac{8}{63} c^{(27)}\right)\left(T_{3}^{2}+\frac{1}{4}\right)+c^{(1)}, \\
& M_{\Omega^{-}}^{\mathrm{EM}}=-\frac{1}{4}\left(c^{(8)}-\frac{4}{21} c^{(27)}\right)+c^{(1)},
\end{aligned}
$$

respectively. Since the center of baryon masses can absorb the singlet contributions to the EM masses with $c^{(1)}$, we can safely neglect them for EM mass differences. Moreover, they are not pertinent to the EM mass differences in which they are canceled out. Therefore, the expressions of the EM mass differences of SU(3) baryons have only two unknown parameters, i.e. $c^{(8)}$ and $c^{(27)}$. As shown in Eqs.(2·18, 2.19), they are expressed in terms of the isospin third component $T_{3}$, its square $T_{3}^{2}$, and the constant terms arising from the hypercharge. Note that Eqs.(2.18, 2.19) in general 
can be rewritten in terms of the electric charge $Q$ and its square $Q^{2}$. Moreover, it

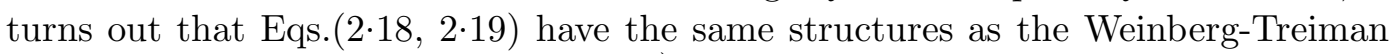
mass formula $M\left(T_{3}\right)=a T_{3}^{2}+b T_{3}+c^{52)}$ expressed in terms of three free parameters $a, b$, and $c$.

In Refs., 44),45) the Dashen ansat $2^{53}$ ) was used for the EM mass splittings of the $\mathrm{SU}(3)$ baryons, which shows $Q^{2}$ proportionality $\left(\left(\Delta M_{B}\right)_{\mathrm{EM}} \sim Q_{B}^{2} M_{B}\right)$. However, this Ansatz was originally used for the squares of $\mathrm{SU}(3)$ pseudoscalar meson masses and is valid only in the chiral limit. In fact, Ref. ${ }^{44}$ employed the Dashen Ansatz and fixed the unknown free parameter appearing in this Ansatz, using the result of $\left(\Sigma^{-}+\Sigma^{+}-2 \Sigma^{0}\right)$ derived in Ref. $\left.{ }^{54}\right)$ However, this Ansatz does not determine the sign of the EM mass splittings. It is straightforward to obtain the EM mass differences for the baryon octet from Eq.(2·18)

$$
\begin{aligned}
\left(M_{p}-M_{n}\right)_{\mathrm{EM}} & =\frac{1}{5}\left(c^{(8)}+\frac{4}{9} c^{(27)}\right), \quad\left(M_{\Sigma^{+}}-M_{\Sigma^{-}}\right)_{\mathrm{EM}}=c^{(8)}, \\
\left(M_{\Xi^{0}}-M_{\Xi^{-}}\right)_{\mathrm{EM}} & =\frac{4}{5}\left(c^{(8)}-\frac{1}{9} c^{(27)}\right) .
\end{aligned}
$$

Using Eq.(2·20), we immediately obtain the following mass formula $c^{(8)}=\left(M_{p}-\right.$ $\left.M_{n}\right)_{\mathrm{EM}}+\left(M_{\Xi^{0}}-M_{\Xi^{-}}\right)_{\mathrm{EM}}=\left(M_{\Sigma^{+}}-M_{\Sigma^{-}}\right)_{\mathrm{EM}}$. This is just the well-known ColemanGlashow mass formula.55) Although these formulae indicate that these three mass differences are dependent on each other, one can adjust the values of the parameters $c^{(8)}$ and $c^{(27)}$ by the method of least squares. In order to determine the parameters $c^{(8)}$ and $c^{(27)}$, we will first use the empirical data estimated in Ref. ${ }^{54}$ Using these empirical and experimental data, we can determine the values of the parameters $c^{(8)}$ and $c^{(27)}$ as follows

$$
c^{(8)}=-0.15 \pm 0.23, \quad c^{(27)}=8.62 \pm 2.39,
$$

in units of $\mathrm{MeV}$ (42)

\subsection{Baryon octet}

The effects of $\mathrm{SU}(3)_{\mathrm{f}}$ and isospin symmetry breakings being taken into account, the mass formulae of the octet are obtained as follows:

$$
\begin{aligned}
M_{N}= & \bar{M}_{\mathbf{8}}+c^{(1)}+\frac{1}{5}\left(c^{(8)}+\frac{4}{9} c^{(27)}\right) T_{3}+\frac{3}{5}\left(c^{(8)}+\frac{2}{27} c^{(27)}\right)\left(T_{3}^{2}+\frac{1}{4}\right) \\
& -\left(m_{d}-m_{u}\right)\left(\delta_{1}-\delta_{2}\right) T_{3}-\left(m_{s}-\hat{m}\right)\left(\delta_{1}+\delta_{2}\right), \\
M_{\Lambda}= & \bar{M}_{\mathbf{8}}+c^{(1)}+\frac{1}{10}\left(c^{(8)}-\frac{2}{3} c^{(27)}\right)-\left(m_{s}-\hat{m}\right) \delta_{2}, \\
M_{\Sigma}= & \bar{M}_{\mathbf{8}}+c^{(1)}+\frac{1}{2} c^{(8)} T_{3}+\frac{2}{9} c^{(27)} T_{3}^{2}-\frac{1}{10}\left(c^{(8)}+\frac{14}{9} c^{(27)}\right) \\
& -\left(m_{d}-m_{u}\right)\left(\delta_{1}+\frac{1}{2} \delta_{2}\right) T_{3}+\left(m_{s}-\hat{m}\right) \delta_{2}, \\
M_{\Xi}= & \bar{M}_{\mathbf{8}}+c^{(1)}+\frac{4}{5}\left(c^{(8)}-\frac{1}{9} c^{(27)}\right) T_{3}-\frac{2}{5}\left(c^{(8)}-\frac{1}{9} c^{(27)}\right)\left(T_{3}^{2}+\frac{1}{4}\right) \\
& -\left(m_{d}-m_{u}\right)\left(\delta_{1}+2 \delta_{2}\right) T_{3}+\left(m_{s}-\hat{m}\right) \delta_{1},
\end{aligned}
$$


where $\delta_{1}$ and $\delta_{2}$ are defined as

$$
\begin{aligned}
\delta_{1} & =-\frac{1}{5} \alpha-\beta+\frac{1}{5} \gamma, \\
\delta_{2} & =-\frac{1}{10} \alpha-\frac{3}{20} \gamma .
\end{aligned}
$$

The center mass $\bar{M}_{\mathbf{8}}$ for the baryon octet from Eq. $(2 \cdot 22)$ is found to be

$$
\bar{M}_{\mathbf{8}}=\frac{1}{3} \bar{M}_{N}+\frac{1}{4} \bar{M}_{\Sigma}+\frac{1}{12} M_{\Sigma^{0}}+\frac{1}{3} \bar{M}_{\Xi}-c^{(1)},
$$

where $\bar{M}_{B}$ indicates the mean values of the masses in the corresponding isospin multiplets, for example, $\bar{M}_{N}=\left(M_{p}+M_{n}\right) / 2$. Note that the $\bar{M}_{\mathbf{8}}$ is expressed in terms of octet masses.

\subsection{Baryon decuplet}

Similarly, we can derive the masses of the baryon decuplet with the center of the decuplet $\bar{M}_{10}$ :

$$
\begin{aligned}
M_{\Delta}= & \bar{M}_{1 \mathbf{0}}+c^{(1)}+\frac{1}{4}\left(c^{(8)}+\frac{8}{63} c^{(27)}\right) T_{3}+\frac{5}{63} c^{(27)} T_{3}^{2}+\frac{1}{8}\left(c^{(8)}-\frac{2}{3} c^{(27)}\right) \\
& -\left(m_{d}-m_{u}\right)\left(\delta_{1}-\frac{3}{4} \delta_{2}\right) T_{3}-\left(m_{s}-\hat{m}\right)\left(\delta_{1}-\frac{3}{4} \delta_{2}\right) \\
M_{\Sigma^{*}}= & \bar{M}_{1 \mathbf{0}}+c^{(1)}+\frac{1}{4}\left(c^{(8)}-\frac{4}{21} c^{(27)}\right) T_{3}+\frac{5}{63} c^{(27)}\left(T_{3}^{2}-1\right) \\
& -\left(m_{d}-m_{u}\right)\left(\delta_{1}-\frac{3}{4} \delta_{2}\right) T_{3}, \\
M_{\Xi^{*}}= & \bar{M}_{\mathbf{1 0}}+c^{(1)}+\frac{1}{4}\left(c^{(8)}-\frac{32}{63} c^{(27)}\right) T_{3}-\frac{1}{4}\left(c^{(8)}+\frac{8}{63} c^{(27)}\right)\left(T_{3}^{2}+\frac{1}{4}\right) \\
& -\left(m_{d}-m_{u}\right)\left(\delta_{1}-\frac{3}{4} \delta_{2}\right) T_{3}+\left(m_{s}-\hat{m}\right)\left(\delta_{1}-\frac{3}{4} \delta_{2}\right), \\
M_{\Omega^{-}}= & \bar{M}_{10}+c^{(1)}-\frac{1}{4}\left(c^{(8)}-\frac{4}{21} c^{(27)}\right)+2\left(m_{s}-\hat{m}\right)\left(\delta_{1}-\frac{3}{4} \delta_{2}\right) .
\end{aligned}
$$

As in the case of the baryon octet, the center of mass splittings $\bar{M}_{10}$ of the baryon decuplet can be expressed as

$$
\bar{M}_{\mathbf{1 0}}=\frac{3}{2} \bar{M}_{\Sigma^{*}}-\frac{1}{2} M_{\Sigma^{* 0}}-c^{(1)} .
$$

Making use of Eq. (2.25), we are able to obtain various mass relations among the decuplet baryons as follows:

$$
\begin{aligned}
M_{\Delta^{++}}-M_{\Delta^{+}} & =\left(M_{\Xi^{* 0}}-M_{\Xi^{*-}}\right)+2 \Delta M_{\Sigma^{*}} \\
M_{\Delta^{+}}-M_{\Delta^{0}} & =M_{\Sigma^{*+}}-M_{\Sigma^{* 0}}=\left(M_{\Xi^{* 0}}-M_{\Xi^{*-}}\right)+\Delta M_{\Sigma^{*}}, \\
M_{\Delta^{0}}-M_{\Delta^{-}} & =M_{\Sigma^{* 0}}-M_{\Sigma^{*-}}=\left(M_{\Xi^{* 0}}-M_{\Xi^{*-}}\right)
\end{aligned}
$$

for $\Delta T_{3}=1$, and

$$
M_{\Delta^{++}}-M_{\Delta^{0}}=2\left(M_{\Xi^{* 0}}-M_{\Xi^{*-}}\right)+3 \Delta M_{\Sigma^{*}}
$$




$$
M_{\Delta^{+}}-M_{\Delta^{-}}=M_{\Sigma^{*+}}-M_{\Sigma^{*-}}=2\left(M_{\Xi^{* 0}}-M_{\Xi^{*-}}\right)+\Delta M_{\Sigma^{*}}
$$

for $\Delta T_{3}=2$. In the case of $\Delta T_{3}=3$, we get

$$
M_{\Delta^{++}}-M_{\Delta^{-}}=3\left(M_{\Xi^{* 0}}-M_{\Xi^{*-}}\right)+3 \Delta M_{\Sigma^{*}} .
$$

In addition, we derive one more mass formula as follows:

$$
2 M_{\Xi^{*-}}-M_{\Sigma^{*-}}=M_{\Omega^{-}} .
$$

Equations (2·27)-(2·30) generalize the Gell-Mann-Okubo mass formulae. While the experimental data for the $\Delta$ isobars are not enough to judge the above-given mass relations, all other relations apart from the $\Delta$ isobars are all in good agreement with the data. If we turn off isospin symmetry breaking, Eq. (2.25) is reduced to the Gell-Mann-Okubo mass formula for the decuplet $\left(\frac{567}{57}\right)$ as follows:

$$
\begin{aligned}
\left(\bar{M}_{\Delta}-\bar{M}_{\Sigma^{*}}\right) & =\left(\bar{M}_{\Sigma^{*}}-\bar{M}_{\Xi^{*}}\right)=\left(\bar{M}_{\Xi^{*}}-\bar{M}_{\Omega^{-}}\right), \\
\bar{M}_{\Delta}-\bar{M}_{\Omega^{-}} & =3\left(\bar{M}_{\Sigma^{*}}-\bar{M}_{\Xi^{*}}\right) .
\end{aligned}
$$

In a $\chi \mathrm{SM}$, it is also possible to connect the mass splittings of the baryon octet to those of the decuplet. (48) Since we include both flavor SU(3) and isospin symmetry breakings, we can derive the following formulae

$$
\begin{aligned}
& 2\left(M_{p}+M_{\Xi^{0}}\right)+3\left(-M_{\Sigma^{*-}}+2 M_{\Xi^{*-}}\right)=3 M_{\Lambda}+2 M_{\Sigma^{+}}-M_{\Sigma^{0}}+3 M_{\Omega^{-}}, \\
& 2\left(M_{n}+M_{\Xi^{-}}\right)+3\left(-M_{\Sigma^{*-}}+2 M_{\Xi^{*-}}\right)=3 M_{\Lambda}+2 M_{\Sigma^{-}}-M_{\Sigma^{0}}+3 M_{\Omega^{-}} .
\end{aligned}
$$

These are the generalization of the Guadagnini mass formula and are deviated from the experimental data by about $4 \mathrm{MeV}$ only, which is remarkable. The basically same formula was obtained in Ref. ${ }^{(58)}$ Using Eq. (2.30) and turning off the effects of isospin symmetry breaking, we reproduce the Guadagnini formula ${ }^{48}$ )

$$
8\left(\bar{M}_{N}+\bar{M}_{\Xi^{*}}\right)+3 \bar{M}_{\Sigma}=11 \bar{M}_{\Lambda}+8 \bar{M}_{\Sigma^{*}} .
$$

\subsection{Baryon antidecuplet}

We now come to the expressions of the mass splittings of the baryon antidecuplet. The masses of the antidecuplet are expressed as

$$
\begin{aligned}
M_{\Theta^{+}}= & \bar{M}_{\overline{\mathbf{1 0}}}+c^{(1)}+\frac{1}{4}\left(c^{(8)}-\frac{4}{21} c^{(27)}\right)-2\left(m_{s}-\hat{m}\right) \delta_{3}, \\
M_{N^{*}}= & \bar{M}_{\overline{\mathbf{1 0}}}+c^{(1)}+\frac{1}{4}\left(c^{(8)}-\frac{32}{63} c^{(27)}\right) T_{3}+\frac{1}{4}\left(c^{(8)}+\frac{8}{63} c^{(27)}\right)\left(T_{3}^{2}+\frac{1}{4}\right) \\
& -\left(m_{d}-m_{u}\right) \delta_{3} T_{3}-\left(m_{s}-\hat{m}\right) \delta_{3}, \\
M_{\Sigma_{\overline{10}}=} & \bar{M}_{\overline{\mathbf{1 0}}}+c^{(1)}+\frac{1}{4}\left(c^{(8)}-\frac{4}{21} c^{(27)}\right) T_{3} \\
& -\frac{5}{63} c^{(27)}\left(T_{3}^{2}-1\right)-\left(m_{d}-m_{u}\right) \delta_{3} T_{3}, \\
M_{\Xi_{3 / 2}^{+}}= & \bar{M}_{\overline{\mathbf{1 0}}}+c^{(1)}+\frac{1}{4}\left(c^{(8)}+\frac{8}{63} c^{(27)}\right) T_{3}-\frac{5}{63} c^{(27)} T_{3}^{2}-\frac{1}{8}\left(c^{(8)}-\frac{2}{3} c^{(27)}\right)
\end{aligned}
$$




$$
-\left(m_{d}-m_{u}\right) \delta_{3} T_{3}+\left(m_{s}-\hat{m}\right) \delta_{3},
$$

where the center of the mass splittings of the baryon antidecuplet is given as

$$
\bar{M}_{\overline{\mathbf{1 0}}}=\frac{3}{2} \bar{M}_{\Sigma_{\overline{10}}}-\frac{1}{2} M_{\Sigma \overline{10}}-c^{(1)}
$$

and $\delta_{3}$ is defined as

$$
\delta_{3}=-\frac{1}{8} \alpha-\beta+\frac{1}{16} \gamma .
$$

Note that while the masses of the baryon octet and decuplet contain $\delta_{1}$ and $\delta_{2}$, those of the antidecuplet include $\delta_{3}$.

Using Eq. (2.34), we can derive similar mass relations to Eqs. $(2 \cdot 22,2 \cdot 25)$ as follows:

$$
\begin{aligned}
& M_{\Xi_{3 / 2}^{+}}-M_{\Xi_{3 / 2}^{0}}=M_{\Sigma_{\overline{10}}^{+}}-M_{\Sigma_{\overline{10}}^{0}}=\left(M_{p^{*}}-M_{n^{*}}\right) \\
& M_{\Xi_{3 / 2}^{0}}-M_{\Xi_{3 / 2}^{-}}=M_{\Sigma_{\overline{10}}^{0}}-M_{\Sigma_{\overline{10}}^{-}}=\left(M_{p^{*}}-M_{n^{*}}\right)-\Delta M_{\Sigma_{\overline{10}}}, \\
& M_{\Xi_{3 / 2}^{-}}-M_{\Xi_{3 / 2}^{--}}=\left(M_{p^{*}}-M_{n^{*}}\right)-2 \Delta M_{\Sigma_{\overline{10}}},
\end{aligned}
$$

for $\Delta T_{3}=1$, and

$$
\begin{aligned}
& M_{\Xi_{3 / 2}^{+}}-M_{\Xi_{3 / 2}^{-}}=M_{\Sigma_{10}^{+}}-M_{\Sigma_{\overline{10}}^{-}}=2\left(M_{p^{*}}-M_{n^{*}}\right)-\Delta M_{\Sigma_{\overline{10}}} \\
& M_{\Xi_{3 / 2}^{0}}-M_{\Xi_{3 / 2}^{--}}=2\left(M_{p^{*}}-M_{n^{*}}\right)-3 \Delta M_{\Sigma_{\overline{10}}}
\end{aligned}
$$

for $\Delta T_{3}=2$. In the case of $\Delta T_{3}=3$, we get

$$
M_{\Xi_{3 / 2}^{+}}-M_{\Xi_{3 / 2}^{--}}=3\left(M_{p^{*}}-M_{n^{*}}\right)-3 \Delta M_{\Sigma_{\overline{10}}},
$$

where $\Delta M_{\Sigma_{\overline{10}}}=M_{\Sigma_{\overline{10}}^{+}}+M_{\Sigma_{\overline{10}}^{-}}-2 M_{\Sigma_{\overline{10}}^{0}}$. The effects of isospin symmetry breaking being switched off, the mass formula of Ref ${ }^{(2)}$ is reproduced as

$$
\bar{M}_{\Theta^{+}}-\bar{M}_{\Xi_{3 / 2}}=3\left(\bar{M}_{N^{*}}-\bar{M}_{\Sigma_{\overline{10}}}\right) .
$$

In addition, we obtain the new mass relations between the baryon octet and antidecuplet:

$$
\left(2 \bar{M}_{N^{*}}+2 \bar{M}_{N}-3 M_{\Lambda}-\bar{M}_{\Sigma_{\overline{10}}}\right)=\left(\bar{M}_{\Sigma}-2 \bar{M}_{\Xi}+M_{\Theta^{+}}\right)
$$

and

$$
3\left(\bar{M}_{\Sigma^{*}}+M_{\Omega^{-}}\right)+2\left(M_{\Theta^{+}}+2 \bar{M}_{\Xi_{3 / 2}}\right)=6\left(\bar{M}_{\Xi^{*}}+\bar{M}_{\Sigma_{\overline{10}}}\right)
$$

between the baryon decuplet and antidecuplet. We also get the mass relation among the baryon octet, decuplet, and antidecuplet

$$
\begin{aligned}
& \left(11 M_{\Lambda}+5 \bar{M}_{\Sigma^{*}}+6 \bar{M}_{\Sigma_{\overline{10}}}\right) \\
= & 3\left(M_{\Omega^{-}}+\bar{M}_{\Sigma}\right)+2\left(M_{\Theta^{+}}+\bar{M}_{\Xi^{*}}\right)+4\left(2 \bar{M}_{N}+\bar{M}_{\Xi_{3 / 2}}\right) .
\end{aligned}
$$




\section{$\S 3 . \quad$ Results and Discussion}

By the least squared method, the model parameters can be adjusted from the studies of mass splittings with the experimental mass values of the baryon octet, $\Omega^{-}(1672)$, and $\Theta^{+}(1524)$ taken as inputs. The effcts of $\mathrm{SU}(3)$ flavor and isospin symmetry breakings are obtained from the baryon octet mass splittings. The masses of $\Omega^{-}(1672)$ and $\Theta^{+}(1524)$ are taken for determination of the mass-splitting centers of the baryon decuplet and antidecuplet, respectively. The centers of mass splittings also can be expressed in terms of the model parameters, the moments of inertia of soliton $I_{1}$ and $I_{2}$ in Eq. (2·10). The Eq. (2.22) yields the ratio of the current light quark masses as follows:

$$
\begin{aligned}
R & =\frac{m_{s}-\hat{m}}{m_{d}-m_{u}} \\
& =\frac{M_{p}-M_{\Sigma^{+}}+M_{\Sigma^{0}}-M_{\Xi^{-}}}{2\left(M_{\Sigma^{+}}-M_{\Sigma^{0}}\right)},
\end{aligned}
$$

which yields

$$
R=58.1 \pm 1.3 \text {, }
$$

Note that in Ref. ${ }^{54}$ ) it is given as $R=43.5 \pm 2.2$, which implies that $R$ in this work

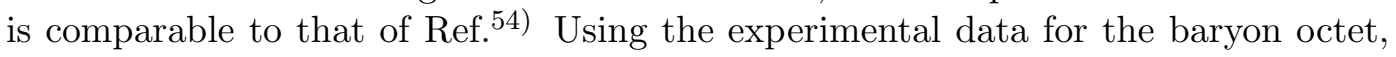
$\Omega$ and $\Theta^{+}$with the value of $R$ in Eq. (3.2), we can determine the mass parameters

$$
\begin{array}{lc}
\left(m_{d}-m_{u}\right) \alpha=-4.390 \pm 0.004, & \left(m_{s}-\hat{m}\right) \alpha=-255.029 \pm 5.821, \\
\left(m_{d}-m_{u}\right) \beta=-2.411 \pm 0.001, & \left(m_{s}-\hat{m}\right) \beta=-140.040 \pm 3.195, \\
\left(m_{d}-m_{u}\right) \gamma=-1.740 \pm 0.006, & \left(m_{s}-\hat{m}\right) \gamma=-101.081 \pm 2.332,
\end{array}
$$

in units of $\mathrm{MeV}$. With these parameters, we find the moments of inertia $I_{1}$ and $I_{2}$. Though $I_{1}$ and $I_{2}$ could be determined by using Eqs.(2.10) and the centers of

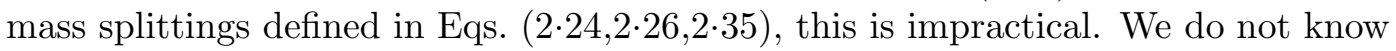
experimentally $M_{\Sigma_{\overline{10}}}$. Thus, in order to determine $I_{1}$ and $I_{2}$ we will rather use the mass formulae for $\Omega^{-}$and $\Theta^{+}$in Eqs.(2.25, 2.34), combining them with the mass parameters determined in Eq. $(3 \cdot 3)$. The obtained values of $I_{1}$ and $I_{2}$ are listed in Table 【.

The mixing coefficients defined in Eq. (2.13) are fixed to be

$$
\begin{aligned}
c_{\overline{10}} & =0.0434 \pm 0.0006, & & c_{27}=0.0203 \pm 0.0003, \\
a_{27} & =0.0903 \pm 0.0013, & & a_{35}=0.0181 \pm 0.0003, \\
d_{8} & =-0.0434 \pm 0.0006, & & d_{27}=0.0365 \pm 0.0005, \\
d_{\overline{35}} & =0.1447 \pm 0.0021 . & &
\end{aligned}
$$

Employing the value of the ratio $\left(m_{d}-m_{u}\right) /\left(m_{d}+m_{u}\right)=0.28 \pm 0.03$, 54 we find the pion-nucleon sigma term unambiguosly:

$$
\Sigma_{\pi N}=(36.4 \pm 3.9) \mathrm{MeV} .
$$

In Ref., $\left.{ }^{2}\right) \Sigma_{\pi N}=45 \mathrm{MeV}$ was used, $\underline{60}$ ) while Ref. $\stackrel{43}{(3)}$ predicted $\Sigma_{\pi N}=73 \mathrm{MeV}$ in studying the baryon antidecuplet.61), (62) In fact, larger values of the $\Sigma_{\pi N}$ are 
Table I. The comparison of the present results of important parameters with those of other works. The masses of the baryon antidecuplet members used as input are listed in the second row. Note that the results listed in the final column do not contain the isospin symmetry breaking effects, so that they are slightly different from those of Eq.(3.3).

\begin{tabular}{c|c|c|c|c}
\hline & Diakonov et al. & Ellis et al. $^{[3]}$ & $\chi \mathrm{QSM}^{[59}$ & This work \\
\hline $\begin{array}{c}\text { Input } \\
\text { masses }\end{array}$ & $N^{*}(1710 \mathrm{MeV})$ & $\Theta^{+}(1539 \pm 2 \mathrm{MeV})$ & $\cdots$ & $\Theta^{+}(1524 \pm 5 \mathrm{MeV})$ \\
\hline$\Sigma_{\pi N}$ & $45 \mathrm{MeV}^{\star}$ & $73 \mathrm{MeV}$ & $41 \mathrm{MeV}$ & $36.4 \pm 3.9 \mathrm{MeV}$ \\
\hline$I_{1}$ & $1.29 \mathrm{fm}$ & $1.27 \mathrm{fm}$ & $1.06 \mathrm{fm}$ & $1.230 \pm 0.002 \mathrm{fm}$ \\
$I_{2}$ & $0.4 \mathrm{fm}$ & $0.49 \mathrm{fm}$ & $0.48 \mathrm{fm}$ & $0.420 \pm 0.006 \mathrm{fm}$ \\
$m_{s} \alpha$ & $-218 \mathrm{MeV}$ & $-605 \mathrm{MeV}$ & $-197 \mathrm{MeV}$ & $-262.9 \pm 5.9 \mathrm{MeV}$ \\
$m_{s} \beta$ & $-156 \mathrm{MeV}$ & $-23 \mathrm{MeV}$ & $-94 \mathrm{MeV}$ & $-144.3 \pm 3.2 \mathrm{MeV}$ \\
$m_{s} \gamma$ & $-107 \mathrm{MeV}$ & $152 \mathrm{MeV}$ & $-53 \mathrm{MeV}$ & $-104.2 \pm 2.4 \mathrm{MeV}$ \\
$c_{\overline{10}}$ & 0.084 & 0.088 & 0.037 & $0.0434 \pm 0.0006$ \\
\hline \multicolumn{4}{|r}{}
\end{tabular}

predicted to describe the mass splitting in the baryon antidecuplet 62 Indeed, the larger value of the $\Sigma_{\pi N}$ reduces the antidecuplet splitting noticeably. (20) In Ref., 62 ) the $\Sigma_{\pi N}$ has been extracted by using the $\Theta^{+}$and $\Xi_{3 / 2}$ masses, based on the $\chi$ QSM: $\Sigma_{\pi N}=(74 \pm 12) \mathrm{MeV}$. However, the present result of $\Sigma_{\pi N}$ remains rather smaller than the previous analyses based on the masses of the baryon antidecuplet.

In Table I, we compare the present results of the important parameters with those of other works. Note that Ref. ${ }^{2}$ ) use the $\pi N$ sigma term as input, while in the present work we are able to predict its value, since we have considered the effects of isospin symmetry breaking. The predicted value of $\Sigma_{\pi N}$ in this work is different from that of the recent calculation in the $\chi \mathrm{QSM}$, which is listed in the fourth column. The results of moments of inertia $I_{1}$ and $I_{2}$ are comparable to those of Refs. (2),43) However, the important parameters $\alpha, \beta$, and $\gamma$ turn out to be rather different. Even the sign of $\gamma$ in Ref. ${ }^{43}$ ) is different from the present result. The result of the mixing parameter $c_{\overline{10}}$ turns out to be almost two times less than those of Refs. ${ }^{2}$, (43) while it is comparable to that of the $\chi$ QSM. This parameter is of great importance to determine the coupling constants for the $K^{*} N \Theta^{+}$vertex $\left.{ }^{63}\right)$ For example, if we use the values of $c_{\overline{10}}$ in Refs. ${ }^{2}$, ,43) the vector coupling constant $g_{K^{*} n \Theta}\left(=f_{K^{*}} \sqrt{15} c_{\overline{10}}\right)$ yields about 1.86 and 1.95 , respectively, whereas the present value produces $g_{K^{*} n \Theta}=0.96$. We want to mention that the measurement of the $\Theta^{+}$photoproduction prefers smaller values of $g_{K^{*} n} \Theta^{\frac{15}{15}}$ The detailed analysis of this coupling constant will appear elsewhere.

It is worthwhile to compare closely the present results for the predicted values of $M_{\Theta^{+}}$and $M_{N^{*}}$ with those of Refs. ${ }^{21,43}$.43 For this comparison, let us turn off the effects of isospin symmetry breaking. Then, the only relevant parameter is $m_{\mathrm{s}} \delta_{3}$ for the masses of the baryon antidecuplet, as shown in Eq. (2.34). The corresponding results are obtained, respectively, as $m_{\mathrm{s}} \delta_{3}=177 \mathrm{MeV}$ (Diakonov et al.), $m_{\mathrm{s}} \delta_{3}=108$ $\mathrm{MeV}$ (Ellis et al.), and $m_{\mathrm{s}} \delta_{3}=171 \mathrm{MeV}$ from the present work. Using the mass of $\Theta^{+}$measured by the LEPS collaboration, i.e. $M_{\Theta^{+}}=1524 \mathrm{MeV}$, one finds the $N^{*}$ masses, respectively, as follows: $M_{N^{*}}=1700 \mathrm{MeV}$ (Diakonov et al.), $M_{N^{*}}=1631$ $\mathrm{MeV}$ (Ellis et al.), $M_{N^{*}}=1694 \mathrm{MeV}$ for this work. The experimental data for the 
$N^{*}$ mass $M_{N^{*}}=(1685 \pm 12) \mathrm{MeV}^{\sqrt{28}}$ being considered, the present result turns out to be quite comparable to it. We will show that the results will be improved later with the effects of isospin symmetry breaking switched on.

Table II. Reproduced masses of the baryon octet. The experimental data of octet baryons are taken from the Particle Data Group (PDG).

\begin{tabular}{cccccc}
\hline \multicolumn{2}{c}{ Mass $[\mathrm{MeV}]$} & $T_{3}$ & $Y$ & Exp. [Inputs] & Numerical results \\
\hline \multirow{2}{*}{$M_{N}$} & $p$ & $1 / 2$ & \multirow{2}{*}{1} & $938.27203 \pm 0.00008$ & $938.76 \pm 3.65$ \\
& $n$ & $-1 / 2$ & & $939.56536 \pm 0.00008$ & $940.27 \pm 3.64$ \\
\hline$M_{\Lambda}$ & $\Lambda$ & 0 & 0 & $1115.683 \pm 0.006$ & $1109.61 \pm 0.70$ \\
\hline \multirow{3}{*}{$M_{\Sigma}$} & $\Sigma^{+}$ & 1 & & $1189.37 \pm 0.07$ & $1188.75 \pm 0.70$ \\
& $\Sigma^{0}$ & 0 & \multirow{2}{*}{0} & $1192.642 \pm 0.024$ & $1190.20 \pm 0.77$ \\
& $\Sigma^{-}$ & -1 & & $1197.449 \pm 0.030$ & $1195.48 \pm 0.71$ \\
\hline \multirow{2}{*}{$M_{\Xi}$} & $\Xi^{0}$ & $1 / 2$ & \multirow{2}{*}{-1} & $1314.83 \pm 0.20$ & $1319.30 \pm 3.43$ \\
& $\Xi^{-}$ & $-1 / 2$ & & $1321.31 \pm 0.13$ & $1324.52 \pm 3.44$ \\
\hline
\end{tabular}

In Table II, the reproduced masses of the baryon octet are listed. In Table III. the predicted results of the decuplet masses are listed. Those of the $\Sigma^{*}$ and $\Xi^{*}$ are in remarkable agreement with the data within $0.5 \%$. In Table IV we present the results for the masses of the baryon antidecuplet.

Table III. Predicted masses of the baryon decuplet. The experimental data of decuplet baryons are taken from the Particle Data Group (PDG).

\begin{tabular}{|c|c|c|c|c|c|}
\hline Mass & {$[\mathrm{MeV}]$} & $T_{3}$ & $Y$ & Experiment $^{41}$ & Predictions \\
\hline \multirow{4}{*}{$M_{\Delta}$} & $\Delta^{++}$ & $3 / 2$ & \multirow{4}{*}{1} & \multirow{4}{*}{$1231-1233$} & $1248.54 \pm 3.39$ \\
\hline & $\Delta^{+}$ & $1 / 2$ & & & $1249.36 \pm 3.37$ \\
\hline & $\Delta^{0}$ & $-1 / 2$ & & & $1251.53 \pm 3.38$ \\
\hline & $\Delta^{-}$ & $-3 / 2$ & & & $1255.08 \pm 3.37$ \\
\hline \multirow{3}{*}{$M_{\Sigma^{*}}$} & $\Sigma^{*+}$ & 1 & \multirow{3}{*}{0} & $1382.8 \pm 0.4$ & $1388.48 \pm 0.34$ \\
\hline & $\Sigma^{* 0}$ & 0 & & $1383.7 \pm 1.0$ & $1390.66 \pm 0.37$ \\
\hline & $\Sigma^{*-}$ & -1 & & $1387.2 \pm 0.5$ & $1394.20 \pm 0.34$ \\
\hline \multirow{2}{*}{$M_{\Xi * 0}$} & $\Xi^{* 0}$ & $1 / 2$ & \multirow{2}{*}{-1} & $1531.80 \pm 0.32$ & $1529.78 \pm 3.38$ \\
\hline & $\Xi^{*-}$ & $-1 / 2$ & & $1535.0 \pm 0.6$ & $1533.33 \pm 3.37$ \\
\hline$M_{\Omega^{-}}^{\star}$ & $\Omega^{-}$ & 0 & -2 & $1672.45 \pm 0.29$ & Input \\
\hline
\end{tabular}

Table IV. Predicted masses of the baryon antidecuplet.

\begin{tabular}{cccccc}
\hline \multicolumn{2}{c}{ Mass } & $T_{3}$ & $Y$ & Experiment & Predictions \\
\hline$M_{\Theta+}$ & $\Theta^{+}$ & 0 & 2 & $1524 \pm 55^{[5]}$ & Input \\
\hline \multirow{2}{*}{$M_{N^{*}}$} & $p^{*}$ & $1 / 2$ & 1 & \multirow{2}{*}{$1686 \pm 12^{(28)}$} & $1688.18 \pm 10.53$ \\
& $n^{*}$ & $-1 / 2$ & & & $1692.16 \pm 10.53$ \\
\hline \multirow{3}{*}{$M_{\Sigma_{\overline{10}}}$} & $\Sigma_{\overline{10}}^{+}$ & 1 & & & $1852.35 \pm 10.00$ \\
& $\Sigma_{\overline{10}}^{0}$ & 0 & 0 & & $1856.33 \pm 10.00$ \\
& $\Sigma_{\overline{10}}^{-}$ & -1 & & & $1858.95 \pm 10.00$ \\
\hline \multirow{4}{*}{$M_{\Xi_{3 / 2}}$} & $\Xi_{3 / 2}^{+}$ & $3 / 2$ & & & $2016.53 \pm 10.53$ \\
& $\Xi_{3 / 2}^{0}$ & $1 / 2$ & -1 & & $2020.51 \pm 10.53$ \\
& $\Xi_{3 / 2}^{-}$ & $-1 / 2$ & & & $2023.12 \pm 10.53$ \\
& $\Xi_{3 / 2}^{-}$ & $-3 / 2$ & & & $2024.37 \pm 10.53$ \\
\hline
\end{tabular}


In this work, we use the mass of the $\Theta^{+}$taken from Ref. ${ }^{[15}$ ) as input. Though the NA49 data of $\Xi_{3 / 2}^{--}(1862){ }^{64)}$ is still under debate, we can compare the present results with that. As shown in Table IV, the results seem to be quite larger than the NA49 data. Note that, however, A recent analysis of Ref. ${ }^{65}$ ) yields the mass ranges of $\Sigma_{\overline{10}}$ and $\Xi_{3 / 2}$ as

$$
M_{\Sigma_{\overline{10}}}=1795 \mathrm{MeV}-1830 \mathrm{MeV}, \quad M_{\Xi_{3 / 2}}=1900 \mathrm{MeV}-1970 \mathrm{MeV},
$$

which are comparable to the present results.

\section{$\S 4$. Summary and conclusion}

In the present work, we have investigated the mass splittings of the $\mathrm{SU}(3)$ baryons within the framework of an $\mathrm{SU}(3)$ chiral soliton model, taking into account $\mathrm{SU}(3)$ and isospin symmetry breakings due to the electromagnetic self-interactions as well as hadronic isospin mass differences. We found various mass relations of the baryon octet, decuplet, and antidecuplet. In particular, we obtained the generalized Gell-Mann-Okubo mass formulae that are well satisfied with the experimental data. We also derived the Coleman-Glashow mass formula and Guadagnini mass relation. In addition, similar mass relations in the baryon antidecuplet were presented.

In order to determine the unknown model parameters $\alpha, \beta$, and $\gamma$, we employed the existing experimental data for the baryon octet, the $\Omega^{-}$, and the $\Theta^{+}$. We then performed the minimization of the $\chi^{2}$. The second moment of inertia $I_{2}$ was also found, which is an essential key to explain the mass splittings within the baryon antidecuplet. Moreover, the pion-nucleon sigma term was determined to be $\Sigma_{\pi N}=$ $(36.4 \pm 3.9) \mathrm{MeV}$. The present results of the $\Sigma^{*}$ and $\Xi^{*}$ masses were in remarkable agreement with the experimental data. It indicates that the mass of the $\Theta^{+}$used as input in the present scheme is rather compatible with existing experimental data for the baryon octet and decuplet.

The present work is distinguished from the previous studies2),43) based on the chiral soliton model, which also deal with the mass splittings of the $\mathrm{SU}(3)$ baryons. The second moment of inertia $I_{2}$ plays a crucial role in explaining the heavier masses of the baryon antidecuplet, compared to those of the octet and decuplet. However, it was not possible to fix it unambiguously in previous works, so that results of the model calculations had to be used. Moreover, since the $\Sigma_{\pi N}$ was not uniquely known empirically, some ambiguities were inevitable in previous analyses. While Refs. ${ }^{2}$, (43) used the experimental data for the baryon octet, they did not consider isospin symmetry breaking, so that they were unable to incorporate whole experimental information.

In the present work, we were able to fix all model parameters by using the experimental data for the masses of the baryon octet and parts of the baryon decuplet and antidecuplet, because effects of isospin symmetry breaking have been fully taken into account. Thus, we have produced the masses of the baryon antidecuplet as well as of the decuplet without any further adjustable parameter.

While we determined the masses of the baryon decuplet and antidecuplet, we have not considered in the present work the corresponding decay widths which are 
very important to understand those baryons. In the previous works in the chiral soliton models, one of the parameters for the decay width of the $\Theta^{+}$has been taken from the model calculations. Moreover, the effects of $\mathrm{SU}(3)$ symmetry breaking have never been fully considered. In order to calculate the widths of the antidecuplet systematically, we have to fix all relevant parameters, using the experimental data for axial-vector constants as well as hyperon semileptonic decays. The corresponding investigation is under way.

\section{Acknowledgments}

The authors are grateful to the late Klaus Goeke for his support and contribution to the early stage of the present work. They are also thankful to M.V. Polyakov for his interest in this work and constructive suggestions. They express their gratitude to J. Franklin for constructive criticism of various mass relations. Gh.-S. Yang expresses his gratitude to M. Praszałowicz and S. i. Nam for valuable discussions and comments. The present work is supported by Inha University Research Grant.

\section{References}

1) M. Praszałowicz, in Proceedings of the Workshop on Skyrmions and Anomalies, Krakow, Poland, 1987, Eds. M. Jezabek and M. Praszałowicz, (World Scientific, Singapore, 1987).

2) D. Diakonov, V. Petrov, and M. V. Polyakov, Z. Phys. A 359 (1997), 305.

3) M. Praszalowicz, Phys. Lett. B 575 (2003), 234.

4) T. Nakano et al. [LEPS Collaboration], Phys. Rev. Lett. 91(2003), 012002.

5) M. Battaglieri et al. [CLAS Collaboration], Phys. Rev. Lett. 96 (2006), 042001.

6) B. McKinnon et al. [CLAS Collaboration], Phys. Rev. Lett. 96 (2006), 212001.

7) S. Niccolai et al. [CLAS Collaboration], Phys. Rev. Lett. 97 (2006), 032001.

8) R. De Vita et al. [CLAS Collaboration], Phys. Rev. D 74 (2006), 032001.

9) V. V. Barmin et al. [DIANA Collaboration], Phys. Atom. Nucl. 70 (2007), 35.

10) V. V. Barmin et al. [DIANA Collaboration], Phys. Atom. Nucl. 73 (2010), 1168.

11) A. Aleev and [SVD Collaboration], hep-ex/0509033.

12) T. Hotta [LEPS Collaboration], Acta Phys. Polon. B 36 (2005), 2173.

13) K. Miwa et al. [KEK-PS E522 Collaboration], Phys. Lett. B 635 (2006), 72.

14) A. Aleev et al. [SVD Collaboration], arXiv:0803.3313 [hep-ex]

15) T. Nakano et al. [LEPS Collaboration], Phys. Rev. C 79 (2009), 025210.

16) V. Kuznetsov [GRAAL Collaboration], arXiv:hep-ex/0409032.

17) V. Kuznetsov et al., arXiv:hep-ex/0601002.

18) V. Kuznetsov [GRAAL Collaboration], Phys. Lett. B 647 (2007), 23.

19) A. Fix, L. Tiator and M. V. Polyakov, Eur. Phys. J. A 32 (2007), 311.

20) D. Diakonov and V. Petrov, Phys. Rev. D 69 (2004), 094011.

21) R. A. Arndt, Y. I. Azimov, M. V. Polyakov, I. I. Strakovsky and R. L. Workman, Phys. Rev. C 69 (2004), 035208.

22) M. V. Polyakov and A. Rathke, Eur. Phys. J. A 18 (2003), 691.

23) H.-Ch. Kim, M. Polyakov, M. Praszałowicz, G. S. Yang and K. Goeke, Phys. Rev. D 71 (2005), 094023.

24) V. Kuznetsov, M. Polyakov, T. Boiko, J. Jang, A. Kim, W. Kim and A. Ni, arXiv:hepex/0703003.

25) V. Kuznetsov et al., arXiv:0801.0778 [hep-ex].

26) V. Kuznetsov et al., arXiv:1003.4585 [hep-ex].

27) V. Kuznetsov et al., Acta Phys. Polon. B 39 (2008), 1949.

28) V. Kuznetsov and M. V. Polyakov, JETP Lett. 88 (2008), 347 [arXiv:0807.3217 [hep-ph]].

29) O. Bartalini et al. [The GRAAL collaboration], Eur. Phys. J. A 33 (2007), 169.

30) D. Elsner and [CBELSA Collaboration], Eur. Phys. J. A33 (2007), 147.

31) Y. Azimov, V. Kuznetsov, M. V. Polyakov and I. Strakovsky, Eur. Phys. J. A 25 (2005), 
325.

32) K. S. Choi, S. i. Nam, A. Hosaka and H.-Ch. Kim, Phys. Lett. B 636 (2006), 253.

33) K. S. Choi, S. i. Nam, A. Hosaka and H.-Ch. Kim, Jour. Phys. G 36 (2009), 015008.

34) I.I. Strakovsky, R.A. Arndt, Ya.I. Azimov, M.V. Polyakov and R.L. Workman, AIP Conf. Proc., 775 (2005), 41.

35) C. V. Christov, A. Blotz, K. Goeke, P. Pobylitsa, V. Petrov, M. Wakamatsu and T. Watabe, Phys. Lett. B 325 (1994), 467.

36) A. Blotz, M. Praszałowicz and K. Goeke, Phys. Rev. D 53 (1996), 485.

37) A. Blotz, K.Goeke, N. W. Park, D. Diakonov, V. Petrov and P. V. Pobylitsa, Phys. Lett. B 287 (1992), 29.

38) A. Blotz, D. Diakonov, K. Goeke, N. W. Park, V. Petrov and P. V. Pobylitsa, Nucl. Phys. A 555 (1993), 765.

39) H. Walliser, in Proceedings of the Workshop on Baryons as Skyrme Solitons, Siegen, Germany, 1992, Ed. G. Holzwarth (World Scientific, Singapore, 1992).

40) H. Walliser, Nucl. Phys. A 548 (1992), 649.

41) K. Nakamura et al. [Particle Data Group], J. Phys. G 37 (2010), 075021.

$42)$ G. S. Yang, H.-Ch. Kim and M. V. Polyakov, [arXiv:1009.5250 [hep-ph]], Phys. Lett. B. (2010) in press.

43) J. R. Ellis, M. Karliner and M. Praszalowicz, JHEP 0405 (2004), 002.

44) M. Praszałowicz, A. Blotz and K. Goeke, Phys. Rev. D 47 (1993), 1127.

45) A. Blotz, K. Goeke and M. Praszałowicz, Acta Phys. Polon. B 25 (1994), 1443.

46) C. V. Christov et al.,Prog. Part. Nucl. Phys. 37 (1996), 91.

47) E. Witten, Nucl. Phys. B 223 (1983), 433.

48) E. Guadagnini, Nucl. Phys . B 236 (1984), 35.

49) S. Jain and S. R. Wadia, Nucl. Phys. B 258 (1985), 713.

50) P. V. Pobylitsa, E. Ruiz Arriola, T. Meissner, F. Grummer, K. Goeke and W. Broniowski, J.Phys. G 18 (1992), 1455.

51) H.-Ch. Kim, M. Praszałowicz, M. V. Polyakov and K. Goeke, Phys. Rev. D 58 (1998), 114027.

52) S. Weinberg and S. B. Treiman, Phys. Rev. 116 (1959), 465.

53) R. Dashen, Phys. Rev. 183 (1969), 1245.

54) J. Gasser and H. Leutwyler, Phys. Rept. 87 (1982), 77.

55) S. R. Coleman and S. L. Glashow, Phys. Rev. Lett. 6 (1961), 423.

56) M. Gell-Mann, Phys. Rev. 125 (1962), 1067.

57) S. Okubo, Prog. Theor. Phys. 27 (1962), 949.

$58)$ G. Morpurgo, Phys. Rev. Lett. 68 (1992), 139

$59)$ T. Ledwig, H.-Ch. Kim and K. Goeke, Phys. Rev. D 78 (2008), 054005.

60) J. Gasser, H. Leutwyler and M. E. Sainio, Phys. Lett. B 253 (1991), 252.

61) M. M. Pavan, I. I. Strakovsky, R. L. Workman and R. A. Arndt, PiN Newslett. 16 (2002), 110.

$62)$ P. Schweitzer, Eur. Phys. J. A 22 (2004), 89

63) T. Ledwig, H. C. Kim and K. Goeke, Nucl. Phys. A 811 (2008), 353.

64) C. Alt et al. [NA49 Collaboration], Phys. Rev. Lett. 92 (2004), 042003.

65) K. Goeke, M. V. Polyakov and M. Praszałowicz, [arXiv:0912.0469 [hep-ph]]. 